Europhys. Lett., 68 (4), pp. 582-588 (2004)

DOI: $10.1209 / \mathrm{epl} / \mathrm{i} 2004-10230-6$

\title{
The magnetic structure of $\mathrm{Fe}_{78} \mathrm{Si}_{9} \mathrm{~B}_{13}$ commercial metallic glasses
}

\author{
A. R. Wildes ${ }^{1}$, N. Cowlam ${ }^{2}$, Q. A. Pankhurst ${ }^{3}$, \\ S. Al-Heniti ${ }^{4}$ and M. R. J. GiBBS ${ }^{2}$ \\ 1 Institut Laue-Langevin - BP 156, 38042 Grenoble Cédex 9, France \\ 2 Department of Physics and Astronomy \\ University of Sheffield - Sheffield S3 7RH, UK \\ 3 London Centre for Nanotechnology and Department of Physics and Astronomy \\ University College London - London WC1E 6BT, UK \\ 4 Department of Physics, King Abdulaziz University - Jeddah 21589, Saudi Arabia
}

received 12 February 2004; accepted in final form 20 September 2004 published online 20 October 2004

PACS. 75.50.Kj - Amorphous and quasicrystalline magnetic materials.

PACS. 75.50. Bb - Fe and its alloys.

PACS. 25.40.Dn - Elastic neutron scattering.

\begin{abstract}
X-ray and polarized neutron scattering techniques have been used to examine the magnetic structure of wide-ribbon $\mathrm{Fe}_{78} \mathrm{Si}_{9} \mathrm{~B}_{13}$ commercial metallic glass (METGLAS@ 2605S2). Samples with well-defined geometry have been made for the experiments at $300 \mathrm{~K}$ and in $1.1 \mathrm{~T}$ and have been measured in the As-received, Field Annealed and Stress Relieved states. The data show that all three samples are spatially correlated non-collinear ferromagnets. A new method of analysis has been applied to show that the non-collinear components of the moments are correlated over several neighbour spacings, $\approx 50 \%$ of the range of the atomic correlations, and that, while annealing treatments do not have a profound effect on the correlations, the non-collinear components are larger in the annealed samples.
\end{abstract}

Ferromagnetic iron-metalloid (P, Si, B) metallic-glass alloys are magnetically extremely soft. They are therefore produced commercially for use in a variety of applications such as magnetic sensors, security tags, and transformer materials. The alloys display anomalous behaviour linked to their magnetism, leading to the discovery that their magnetic structure may be non-collinear. Experimental evidence to support non-collinear ferromagnetism in iron-metalloid glasses has come from techniques such as magnetostriction [1], Mössbauer spectroscopy [2-4], high-field susceptibility [5], and neutron scattering with polarization analysis [6]. The discovery of non-collinear structures has stimulated theory developments, including powerful computing methods to calculate the magnetic properties of metallic glasses. For example, ab initio band-structure calculations have shown that non-collinear ferromagnetic structures can be energetically favourable for (FeNi)B metallic glasses [7]. Amorphous iron magnetism has therefore re-emerged as a topic of considerable current interest.

$\mathrm{Fe}_{78} \mathrm{Si}_{9} \mathrm{~B}_{13}$ (METGLAS $囚 2605-\mathrm{S} 2$ ) is a commercially produced, chemically simple ironmetalloid metallic glass. The system has been extensively studied, with recent measurements (C) EDP Sciences 
using Mössbauer spectroscopy [3,4] showing that non-collinear ferromagnetic structures may exist in this system and that the structure may depend upon the heat treatment of the sample. Models for magnetic structure have been proposed [3], but could not be confirmed. We have therefore applied the techniques of neutron scattering with polarization analysis and X-ray diffraction to determine the magnetic structure, and the changes on heat treatment.

Polarization-dependent neutron scattering from commercial $\mathrm{Fe}_{78} \mathrm{Si}_{9} \mathrm{~B}_{13}$ metallic-glass ribbons has been measured in the past, concluding that the samples were collinear ferromagnets [6]. The quality of that data was not exceptional, and only two of the four polarization states were measured, leaving the analysis prone to possible systematic error. A reexamination of the system is timely following dramatic improvements in instrumentation and analysis software and the conclusions of the other studies $[3,8]$. The availability of wide-ribbon samples also allowed for an improved sample geometry. The large width minimized demagnetization due to edge effects and ensured that the samples were of even density with well-defined dimensions, avoiding potential systematic errors and facilitating the analysis. The quality control implicit in a commercial material also suggests that the samples were free from gross compositional inhomogeneities that can affect laboratory grown samples [9].

The scattering cross-sections for a multicomponent glassy system depend on the atomic pair number density $\rho_{i j}(r)$ and the scattering amplitudes of the species $\phi_{i, j}$ via the equation

$$
\frac{\partial \sigma}{\partial \Omega}=\sum_{i, j} \phi_{i} \phi_{j} \int_{0}^{\infty} 4 \pi r^{2}\left(\rho_{i j}(r)-\rho_{0 j}\right) \frac{\sin Q r}{Q r} \mathrm{~d} r .
$$

X-ray scattering, with $\phi=\left(e^{2} / m_{e}\right) f_{X}(Q)$, where $f_{X}(Q)$ is the atomic form factor, probes the atomic/chemical structure. Neutron scattering is sensitive to the magnetic structure and, when used with polarization analysis, can discriminate between collinear and non-collinear ferromagnetic order. When the neutron polarization is perpendicular to the scattering vector, the polarization is unchanged on scattering from the nucleus and the collinear component of the magnetic moment, $\mu_{\|}$, and is called non-spin flip scattering, $\partial \sigma^{\mathrm{nsf}} / \partial \Omega$. The scattering amplitude is written $\phi=b \pm\left(\gamma e^{2} / m_{e}\right) f_{\mathrm{m}}(Q) \mu_{\|}$, where $b$ is the nuclear scattering length [10] and $f_{\mathrm{m}}(Q)$ is the magnetic form factor [11]. The sign depends on whether the polarization is parallel or antiparallel to $\mu_{\|}$. Scattering from non-collinear components of the magnetic moments, $\mu_{\perp}$, causes the polarization to invert and is called spin flip scattering, $\partial \sigma^{\mathrm{sf}} / \partial \Omega$, with amplitude $\phi=1 / \sqrt{ } 2\left(\gamma e^{2} / m_{e}\right) f_{\mathrm{m}}(Q) \mu_{\perp}$. A finite, coherent spin flip cross-section is therefore direct evidence for non-collinearity in the magnetic structure.

Three sets of samples were cut from a roll of the commercial "ultra-wide" continuously cast ribbon, METGLAS®2605-S2, supplied by Allied Chemical Corporation. Two of the sets were heat-treated in a furnace at $400^{\circ} \mathrm{C}$ for 40 minutes. One set, hereafter called Field Annealed, was subjected to a magnetic field of $\approx 0.09 \mathrm{~T}$ during the treatment, applied with permanent magnets in the plane of the sample. The other heat-treated sample will be called Stress Relieved, with the third measured As-received. The X-ray measurements were performed at the King Abdulaziz University using Mo $K_{\alpha}$ radiation, $\lambda=0.71073 \AA$. All the diffraction patterns were very similar, an example of which is shown in fig. 1. The absence of Bragg peaks shows that the heat treatments caused no crystallization. The neutron data were collected with the IN20 polarized neutron three-axis spectrometer at the Institut Laue-Langevin. A ferromagnet must be monodomain for polarized neutron experiments, otherwise the neutrons will be randomly depolarized and any information on the magnetic structure will be scrambled. Consequently, the neutron measurements were carried out in a vertical field of $1.1 \mathrm{~T}$ and at $300 \mathrm{~K}$. The samples are known to be monodomain under these conditions [5], which also match a previous study by Mössbauer spectroscopy [4]. The $1.1 \mathrm{~T}$ field was coaxial with the $0.09 \mathrm{~T}$ 


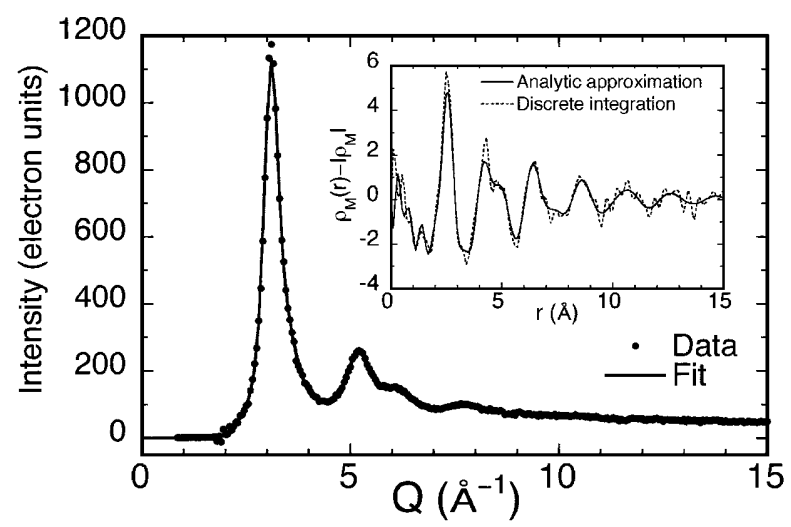

Fig. 1 - The X-ray scattering from the As-received sample. The fit of eq. (4) to the data is also shown. The insert shows a comparison of the global density function as calculated by Fourier-transforming the fit with the function calculated by a discrete Fourier transform of the data.

field used during the heat treatment of the Field Annealed sample. Measurements were made with two incident wave numbers $k=2.662 \AA^{-1}$ and $k=4.1 \AA^{-1}$, representing independent measurements complete with separate backgrounds and instrument calibration. The neutron polarization was determined to be $93 \%$ for $k=2.662 \AA^{-1}$ and $88 \%$ for $k=4.1 \AA^{-1}$. A crystal analyser was used meaning that only the elastic scattering, therefore the static magnetic structure, was measured. The methods used to present the four polarization-dependent crosssections in absolute units have been previously documented in the appendix of ref. [9]. Particular care was taken with attenuation corrections, which were calibrated and cross-checked for each sample using both monochromatic and time-of-flight neutron techniques, and with polarization corrections, which were calibrated and cross-checked using the flipping ratio of the main beam; from a Si (111) Bragg peak; and from scattering from amorphous quartz.

The two non-spin flip cross-sections were averaged for each of the samples, being equivalent to the sum of the cross-section due to nuclear scattering with that due to collinear magnetic order. These cross-sections were similar for the three samples, showing that the data were properly corrected and calibrated. An example of $\left|\partial \sigma^{\mathrm{nsf}} / \partial \Omega\right|$ is given in fig. 2. The dip at $Q \approx 4.4 \AA^{-1}$ is due to a minor overcorrection for an aluminium Bragg peak in the background.

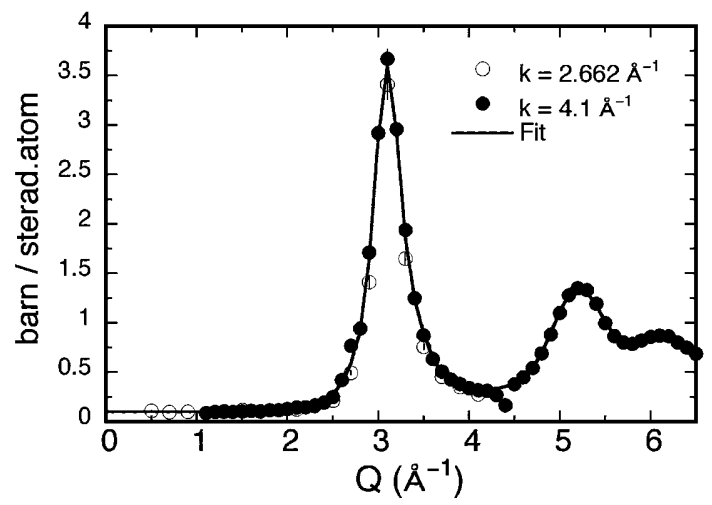

Fig. 2 - The mean non-spin flip scattering, $\left|\partial \sigma^{\text {nsf }} / \partial \Omega\right|$, from the As-received sample, measured at the two wave numbers, with the fit of eq. (4) to the data. 


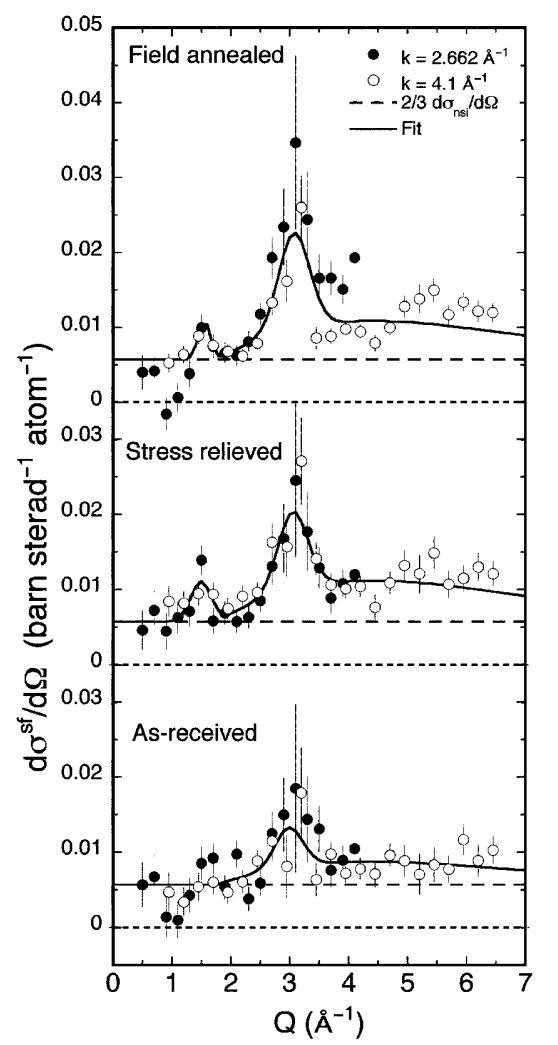

Fig. 3 - The spin flip cross-sections, $\partial \sigma^{\mathrm{sf}} / \partial \Omega$, of the three samples, measured at the two wave numbers, along with the expected incoherent contribution and the fit of eq. (4) to the data.

The spin flip cross-sections, $\partial \sigma^{\mathrm{sf}} / \partial \Omega$, are shown in fig. 3 with the expected incoherent contribution [10]. Although the $\partial \sigma^{\mathrm{sf}} / \partial \Omega$ are $\sim 100$ times smaller than $\left|\partial \sigma^{\text {nsf }} / \partial \Omega\right|$, the data coincide for two independent measurements, which shows that the experiments have been correctly performed and analysed. Each of the $\partial \sigma^{\text {sf }} / \partial \Omega$ shows peaks, confirming that the magnetic structures are non-collinear. The peaks, appearing at the same $Q$ as the first maximum of the glass structure factor, are highly unlikely to be due to depolarization of the neutron beam by the sample as this effect would vary with wavelength, while the $\partial \sigma^{\text {sf }} / \partial \Omega$ in fig. 3 are independent of $k$. Peaks therefore indicate the presence of spatial correlations between the non-collinear moments. There is also possibly a peak at $Q \approx 1.7 \AA^{-1}$. While small, the peak is consistently present in the heat-treated samples and is not visible in the non-spin flip or X-ray data. The peak was therefore regarded as real for subsequent analysis, and as evidence that the correlations differ between the magnetic and chemical structures. The heat treament appears to have influenced the non-collinear structure, with the annealed samples showing larger $\partial \sigma^{\text {sf }} / \partial \Omega$ than the As-received sample. Thus, internal stresses are not the source of the non-collinearity; however, stress may act to suppress the non-collinear state.

None of the cross-sections show any sign of an upturn at small $Q$, which would be evidence for clustering. Any clustering is therefore so large that the associated small-angle scattering is gone at $Q \approx 0.5 \AA^{-1}$, or there is no clustering in these samples in the $1.1 \mathrm{~T}$ applied field. However, the cross-sections may be analysed to determine the magnitude of the non-collinear magnetic components and the spatial correlations between them. Equation (1) must be re- 
arranged to expedite a comparison between the X-ray and neutron data and also to obtain the mean value of $\mu_{\perp}$ from $\partial \sigma^{\mathrm{sf}} / \partial \Omega$. Normalizing eq. (1) to the total scattering level $\left\langle\phi^{2}\right\rangle$ results with

$$
\frac{\partial \sigma}{\partial \Omega}=\left\langle\phi^{2}\right\rangle\left(1+4 \pi \int_{0}^{\infty} r^{2}\left(\rho(r)-\rho_{0}\right) \frac{\sin Q r}{Q r} \mathrm{~d} r\right),
$$

where the global density function

$$
\rho(r)-\rho_{0}=\sum_{\substack{i, j \\ i \neq j}} \frac{\phi_{i} \phi_{j}}{\left\langle\phi^{2}\right\rangle}\left(\rho_{i j}(r)-\rho_{0 j}\right)
$$

contains the atomic pair density functions and their weighting terms based on $\left\langle\phi^{2}\right\rangle$.

The data may therefore be Fourier-transformed to derive the global density function. This is relatively straightforward for the X-ray data, which extends to large $Q$ where the atomic pair number density is constant. The data in figs. 2-3, however, are over a limited range of $Q$, thus a Fourier transform would be subject to aliasing errors. We have therefore adopted a new method for the subsequent analysis. The data was fitted with an expression whose form resembles the cross-section on scattering from an amorphous sample:

$$
\frac{\partial \sigma}{\partial \Omega}=A(Q)\left(1-\exp \left[-\alpha\left(Q-Q_{\mathrm{c}}\right)\right]\right)+\sum_{i} B_{i} \exp \left[-\beta_{i}^{2}\left(Q-Q_{0 i}\right)^{2}\right],
$$

where $A(Q)=0$ for $Q \leq Q_{\mathrm{c}}$ and $A(Q)=\left\langle\phi^{2}\right\rangle$ for $Q>Q_{\mathrm{c}}$. The first term in eq. (4) is a saturating function, approaching $\left\langle\phi^{2}\right\rangle$ as $Q \rightarrow \infty$; the second represents a series of Gaussians, accounting for peaks at positions $Q_{0 i}$. The function can be Fourier-transformed analytically to give a good approximation to $\left(\rho(r)-\rho_{0}\right)$. This approach was tested using the X-ray data. Figure 1 shows a fit of the function to the data and the insert shows a comparison of the global density function estimated from the transform of eq. (4) with a numerical Fourier transform. The level of agreement is satisfactory.

Equation (4) was fitted to the $\left|\partial \sigma^{\text {nsf }} / \partial \Omega\right|$ data, as shown in fig. 2. The limiting $\left\langle\phi^{2}\right\rangle=\left\langle b^{2}\right\rangle$ can be determined from tabulated values [10]. The parameters $Q_{\mathrm{c}}$ and $\alpha$ differed between the fits to the non-spin flip and X-ray data, thus the two parameter sets were fixed for separate fits to the spin flip cross-sections. The fits to $\partial \sigma^{\text {sf }} / \partial \Omega$, shown in fig. 3 were important as $\left\langle\phi^{2}\right\rangle=1 / 2\left(\gamma e^{2} / m_{e}\right)^{2} f_{\mathrm{m}}^{2}(Q)\left\langle\mu_{\perp}^{2}\right\rangle$ gives the mean non-collinear moment per atom. These fits required an appropriate choice of the form factor $f_{\mathrm{m}}(Q)$. The form factors for metallic glasses usually $[6,12]$ decrease more slowly with $Q$ than the listed form factors for metal atoms and their ions [11], so a broadening parameter, $W$, is often included in the analytical approximation for $f_{\mathrm{m}}(Q)[12]$. Previous experiments have found that the $\mathrm{Fe}^{3+}$ form factor, broadened by $W=$ 0.35 , is appropriate for the iron-metalloid glasses [6], and we adopted this form factor for the analysis. Although some of the $\partial \sigma^{\text {sf }} / \partial \Omega$ in fig. 3 show suggestions of peaks at $Q \approx 5.5 \AA^{-1}$, for simplicity and consistency no Gaussians were fitted to the data for $Q>3.5 \AA^{-1}$. This simplification resulted in an overestimation of $\left\langle\mu_{\perp}^{2}\right\rangle$, as introducing Gaussians at larger $Q$ reduced the fitted values of $\left\langle\phi^{2}\right\rangle$; however, the global density functions, being dominated by the shape of the peak at $Q \approx 3 \AA^{-1}$, were approximately correct. The fitted values of $\left\langle\mu_{\perp}^{2}\right\rangle$ are listed in table I.

The values of $\left\langle\mu_{\perp}^{2}\right\rangle$ also depend upon the fixed parameters $Q_{\mathrm{c}}$ and $\alpha$; however, the values in table I confirm that the two annealed samples have a mean non-collinear moment that is larger than the As-received sample. Structural relaxation which accompanies low-temperature annealing decreases the free volume in these glasses [13]; therefore the increase in $\left\langle\mu_{\perp}^{2}\right\rangle$ is consistent with the results of band-structure calculations that show a non-collinear ground 
TABLE I - Table of fitted non-collinear moments for the samples. The paramaters $\alpha$ and $Q_{\mathrm{c}}$ were fixed to 0.32 and 2.00 (from fitting the X-ray data) and 0.18 and 1.77 (from $\left|\partial \sigma^{\mathrm{nsf}} / \partial \Omega\right|$ ), respectively.

\begin{tabular}{|c|c|c|c|c|}
\hline & \multicolumn{2}{|c|}{$\alpha$ and $Q_{\mathrm{c}}$ from X-ray fits } & \multicolumn{2}{|c|}{$\alpha$ and $Q_{\mathrm{c}}$ from non-spin flip fits } \\
\hline Sample & $\left\langle\mu_{\perp}^{2}\right\rangle\left(\mu_{\mathrm{B}}^{2}\right)$ & $\zeta$ & $\left\langle\mu_{\perp}^{2}\right\rangle\left(\mu_{\mathrm{B}}^{2}\right)$ & $\zeta$ \\
\hline Field Annealed & $0.56 \pm 0.07$ & $24 \pm 3$ & $0.74 \pm 0.09$ & $27 \pm 3$ \\
\hline Stress Relieved & $0.57 \pm 0.05$ & $24 \pm 2$ & $0.79 \pm 0.07$ & $28 \pm 2$ \\
\hline As-received & $0.32 \pm 0.06$ & $20 \pm 4$ & $0.43 \pm 0.09$ & $23 \pm 5$ \\
\hline
\end{tabular}

state is preferred as density increases [7]. The increase is qualitatively consistent with previous studies of this system by Mössbauer spectroscopy [3,4]. Although the numerical accuracy is not the most significant aspect of our result, it is nevertheless interesting to compare our results with a particular Mössbauer study [4], also conducted at $300 \mathrm{~K}$ in a field of $1.1 \mathrm{~T}$, which also found evidence for non-collinear ferromagnetism. Three model distributions were proposed to describe the distribution in the angles that the moments made with the mean ferromagnetic axis, but the analysis could not choose between them. The weighted mean angle, $\zeta$, for each of the models was $\approx 10^{\circ}$. A mean angle for the current analysis may be calculated by taking collinear moments from previous high-field magnetization studies, estimated to be $\sim 1.68 \mu_{\mathrm{B}}$ /atom for an annealed sample and $\sim 1.58$ for the As-received [5], and the $\left\langle\mu_{\perp}^{2}\right\rangle$ from table I. The resulting $\zeta$, also listed in table I, are larger than the previous estimates. The differences may be partly due to the assumptions in the analysis of the neutron data, and partly due to subtleties of configurational averaging when comparing the two techniques.

The global density functions, $\rho(r)-\rho_{0}$, derived from the fits of eq. (4) to the data, are shown in fig. 4 . The functions derived from the neutron data have missing Fourier components due to the limited range of $Q$ available for the fits, resulting in large "ripples" for $r<1 \AA$. Otherwise, the derived functions are all very similar. This is as expected for the X-ray and

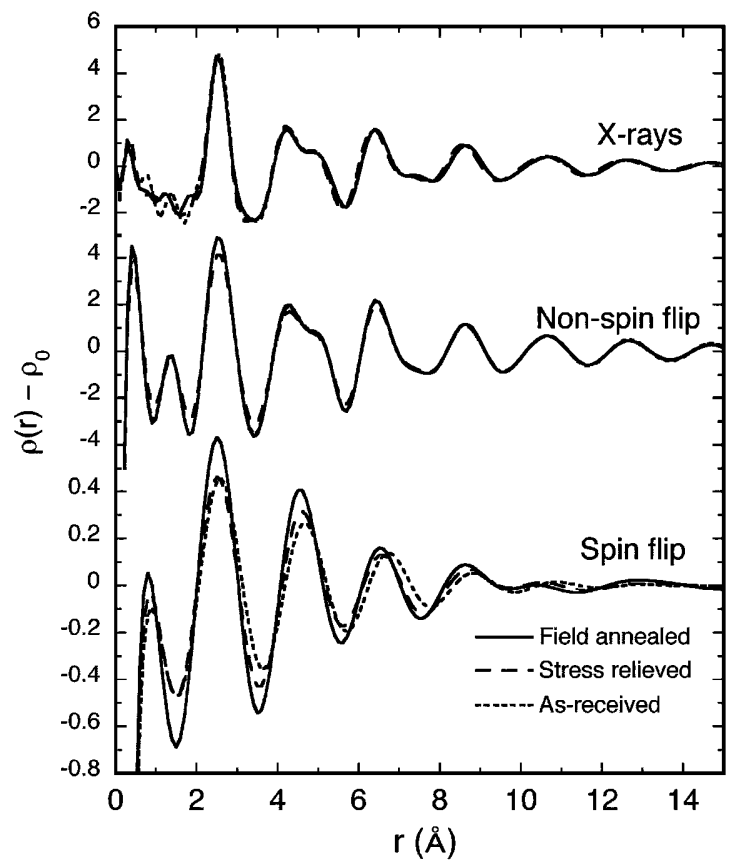

Fig. 4 - The global density functions for the data sets, from the Fourier transforms of the fits to eq. (4). 
non-spin flip measurements, as both are sensitive to the chemical structure of the samples. The functions derived from $\partial \sigma^{\mathrm{sf}} / \partial \Omega$ are also similar, indicating that the nearest-neighbour correlation between the non-collinear components of the moments is ferromagnetic. The range of the correlations is $\approx 8-10 \AA, \approx 50 \%$ the range of the atomic correlations in the glassy structure. It is tempting to look for differences between the non-collinear distributions, and inspection shows that the Field Annealed sample has slightly larger fluctuations indicating a stronger correlation between neighbours. The quality of the data, however, may not support such a detailed examination. If the functions are taken to be equal, heat treatment affects the magnitude of $\left\langle\mu_{\perp}^{2}\right\rangle$ but not the correlation between moments.

In conclusion, these experiments have established that in a field of $1.1 \mathrm{~T}$ the $\mathrm{Fe}_{78} \mathrm{Si}_{9} \mathrm{~B}_{13}$ metallic-glass system has a spatially correlated non-collinear ferromagnetic structure. The global density functions derived from X-ray and polarized neutron data show that the atomic and collinear ferromagnetic structures have similar correlations on similar length scales. The correlations between the non-collinear components of the moments resemble those of the atomic structure over a restricted range.

$$
* * *
$$

The authors would like to thank Prof. P. J. BROwn for a critical reading of the manuscript.

\section{REFERENCES}

[1] Bucholtz F., Koo K. P., Dandridge A. and Sigel G. H., J. Magn. 83 Magn. Mater., 54-57 (1986) 1607.

[2] Melamud M., Swartzendruber L. J., Bennett L. H., Cullen J. and Wun-Fogle M., J. Appl. Phys., 61 (1987) 3644.

[3] Pankhurst Q. A., Jiang J. Z., Betteridge S., Gibbs M. R. J. and Gehring G. A., J. Phys. Condens. Matter, 7 (1995) 9571.

[4] Pankhurst Q. A., Cohen N. S., Fernández Barquín L., Gibbs M. R. J. and Smirnov G. V., J. Non-Cryst. Solids, 287 (2001) 81.

[5] Szymczak P., Graham C. D. jr. and Gibbs M. R. J., IEEE Trans. Magn., 30 (1994) 4788.

[6] Cowley R. A., Patterson C., Cowlam N., Ivison P. K., Martinez J. and Cussen L. D., J. Phys. Condens. Matter, 3 (1991) 9521.

[7] Liebs M. and Fähnle M., J. Phys. Condens. Matter, 8 (1996) 3207.

[8] Pankhurst Q. A. and Gibbs M. R. J., J. Phys. Condens. Matter, 5 (1993) 3275.

[9] Wildes A. R., Stewart J. R., Cowlam N., Al-Heniti S., Kiss L. F. and Kemény T., J. Phys. Condens. Matter, 15 (2003) 675.

[10] Sears V. F., Neutron News., 3 (1992) 26.

[11] Brown P. J., International Tables for Crystallography, edited by Wilson A. J. C., Vol. C (Klewer, Dordrecht) 1995, p. 391.

[12] Bletry J. and Sadoc J. F., J. Phys. F, 5 (1975) L111.

[13] Gibis M. R. J. and Sinning H. R., J. Mater. Sci., 20 (1985) 2517. 